\title{
Association of Killer Cell Immunoglobulin-Like Receptor Genes with Pandemic Influenza A (H1N1)pdm09 Infection in Critically III Macedonian Patients
}

\author{
Aleksandar Petlichkovski ${ }^{1}$, Zvonko Milenkovic $^{2}$, Eli Djulejic ${ }^{3}$, Bisera Jefremovska ${ }^{1}$, Haris Babacic ${ }^{1}$, Ljubomir Ivanovski ${ }^{2}$ Irena Kondova \\ Topuzovska², Katerina Spasovska², Mirko Spiroski ${ }^{*}$ \\ ${ }^{1}$ Institute of Immunobiology and Human Genetics, Faculty of Medicine, University "Ss. Cyril and Methodius", Skopje, Republic of Macedonia; \\ ${ }^{2}$ University Clinic of Infective Diseases, Faculty of Medicine, University “Ss. Cyril and Methodius", Skopje, Republic of Macedonia; ${ }^{3} Q u i n t i l e s$, \\ Belgrade, Republic of Serbia
}

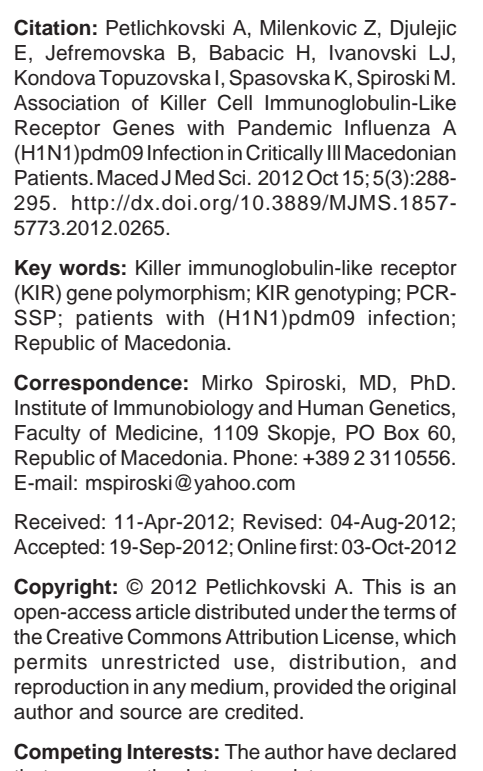
Competing Interests: The author
that no competing interests exist.

\section{Abstract}

Background: Infection by the pandemic influenza A (H1N1)pdm09 virus results in significant pathology disease in many cases in different populations worldwide. The natural killer (NK) cells are among the major effectors important in early innate immune responses to viral infections, interacting with host cells through their activating or inhibiting receptors.

Aim: The aim of this study was to analyze Killer Ig-Like Receptor (KIR) gene polymorphisms in critically ill Macedonian patients with pandemic influenza A (H1N1)pdm09 infection.

Material and Methods: The studied sample consists of 63 critically ill Macedonian patients with pandemic influenza A (H1N1)pdm09 infection. The population genetics analysis package, Arlequin, was used for analysis of the data.

Results: We found that all $16 \mathrm{KIR}$ genes were observed in the studied individuals and framework genes (KIR3DL3, KIR3DP1, KIR2DL4, and KIR3DL2) were present in all individuals. The results of tested linkage disequilibrium (LD) among KIR genes demonstrated that KIR genes present a wide range of linkage disequilibrium. Comparison of KIR gene frequencies between critically ill H1N1/09 Macedonian patients and healthy subjects reveals statistically significant difference for frequency of KIR2DL1 ( $F=1$ in the patients group, and 0.94 in the control group, $p=0.045$ ).

Conclusion: We did not found any significant association of all 16 KIR genes or KIR genotypes with critically ill (H1N1)pdm09 Macedonian patients, except for the KIR2DL1.

\section{Introduction}

Infection with influenza A virus resulted in significant disease in many cases in different populations worldwide. The rapid global spread of the novel swine origin influenza virus A (H1N1)pdm09 and more than 620,000 cases reported worldwide as of the end of 2009 $[1,2]$, prompted the World Health Organization to raise the pandemic alert to the highest level, officially declaring pandemic [3]. An intriguing observation was that the highest prevalence of significant pathology was reported among youths and young adults [4-7]. It is known that innate immune cells, such as the macrophages, neutrophils, and DCs are efficient in clearing inû uenza virus via phagocytosis or promotion of adaptive responses [8-13]. In the recent years, the role of NK cells in response to inû uenza infection has also been studied extensively, and their involvement in the early control of 
inûuenza replication has been in the focus of many research groups [14-21].

The last (H1N1)pdm09 influenza pandemic with swine-origin compared to previous influenza pandemics, appears to have a high infectivity rate but low pathogenicity [22]. So far, despite the extensive research, the host immune factors that might influence the protection of some patients and significant disease in others, have not been clearly established in humans. One of the candidate host factors that might influence the disease severity in the context of (H1N1)pdm09 Influenza infection is the killer immunoglobulin-like receptor (KIR) gene repertoire.

The killer cell immunoglobulin-like receptors are surface molecules found on subsets of lymphoid cells. Most importantly, they influence the natural killer (NK) cells activity in activating or inhibiting manner, depending on the interaction of $K I R$ with HLA molecules present on the target host cells $[23,24]$. The KIR locus contains a family of polymorphic and highly homologous members (14 genes and 2 pseudogenes), which can be activating or inhibitory. Based on the gene content, the haplotypes have been resolved into two broad sets, termed $A$ and $B$ [25]. The different KIR haplotypes vary in the number and type of genes present, but the genes KIR3DL3, KIR3DP1, KIR2DL4 and KIR3DL2 are present on virtually all haplotypes and have therefore been termed framework genes [26]. Population studies performed over the last two decades have revealed extensive diversity at the KIR gene locus, which derives from both, its polygenic and multi-allelic polymorphism, whereas on the basis of gene content, haplotype $\mathrm{B}$ displays a much greater variety of subtypes [27, 28].

Following the model of major histocompatibility complex studies, disease association studies have revealed associations of certain KIR genes and/or genotypes with specific diseases. To date, these studies have mainly targeted viral infections such as human immunodeficiency virus infection, hepatitis $\mathrm{C}$, but also cancer, autoimmune, and inflammatory disorders [2932]. In general, activating genotypes generally appear to be beneficial during viral infections, whereas they increase the risk for susceptibility to autoimmunity [33]. In the present study, we evaluate the association of KIR genes and genotypes with the outcome of human (H1N1)pdm09 Influenza infection.

The aim of this study was to examine KIR gene polymorphisms by determining the frequencies of 16 KIR genes and pseudogenes (KIR2DL1, KIR2DL2,
KIR2DL3, KIR2DL4, KIR2DL5, KIR3DL1, KIR3DL2, KIR3DL3, KIR2DS1, KIR2DS2, KIR2DS3, KIR2DS4, KIR2DS5, KIR3DS1, KIR2DP1, and KIR3DP1) and KIR genotypes in Macedonian patients with pandemic influenza infection, and to compare the gene content between patients with very severe disease including fatalities and patients with less severe disease.

To our knowledge, this is the first study of the diversity of $K I R$ genes in patients with pandemic influenza A (H1N1)pdm09 infection in the Republic of Macedonia and among the few in the world.

\section{Material and Methods}

Population samples. The study included 63 unrelated patients (male 39 and female 25) with laboratory PCR confirmation of Influenza (H1N1)pdm09 pandemic flu hospitalized at the University Clinic of Infective Diseases in Skopje, Republic of Macedonia, between December 2009 and March 2010. Only consecutive patients with severe disease course (with need of ventilation support) were selected.

After signing of written consent, genomic DNA was extracted from the peripheral blood leukocytes using standard phenol/chloroform procedure, described elsewhere [34], and stored in the Macedonian Human DNA Bank (hDNAMKD) [35] until processing.

PCR amplification. For KIR genotyping, commercially available PEL-FREEZ KIR genotyping SSP kit (Dynal Biotech, Brown Deer, WI) was used. It is a PCR-based method (using sequence-specific priming approach) designed to detect the presence or absence of $16 \mathrm{KIR}$ genes and pseudogenes defined by the International nomenclature committee of WHO [36, 37]. Briefly, locus specific primer sets, dispensed in a 96 well thermal tray were used for amplification of genomic DNA. After the amplification, the PCR products are loaded and separated by electrophoresis onto a $2 \%$ agarose gel stained with ethidium bromide, after which the results are interpreted using a worksheet for the specific amplification patterns. The presence of each KIR gene was determined by the presence of a band of DNA of the expected size.

All PCRs contained an internal positive control consisting of an additional pair of primers specific for the growth hormone $(\mathrm{GH})$ gene and a negative control [38]. Individuals were determined negative for a particular KIR gene when a band of expected size was absent in 
the presence of a band for the GH gene. We have used external quality control consisting of cell lines from Immunogenetics and Histocompatibility Worskshop Conferences and Centre d' Etude du Polymorphisme Humain.

Statistical analysis. The occurrence of KIR genes in individuals (frequency $=F$ ) was obtained by direct counting. Gene frequencies (GF) were calculated using the formula $G F=1-\sqrt{ }(1-F)$, being aware of the limitation in its ability to detect KIR genes present at low frequency. For analysis of the molecular polymorphism of the locus studied, the Arlequin software version 3.0 (Genetics and Biometry Laboratory, University of Geneva, Switzerland) [39] was used.

Linkage disequilibrium (LD) values for two locus associations were calculated using $2 \times 2$ tables [40]. Because LD is not independent of allele frequencies, normalized LD was calculated as described previously $[41,42]$. Comparisons of different genotypes for two groups were tested by the $\chi 2$ test. Crude odds ratios (OR) were calculated within $95 \% \mathrm{Cl}$.

\section{Results}

KIR gene frequencies. The frequencies of the 16 KIR genes (14 genes and 2 pseudogenes) determined in the 63 Macedonian (H1N1)pdm09 patients, is shown in Table 1 along with the corresponding frequencies of the 214 healthy Macedonian controls. All 16 KIR genes were observed in both groups of the studied population and framework genes (KIR3DL3, KIR3DP1, KIR2DL4, and $K I R 3 D L 2)$ were present in all individuals.
Comparison of KIR gene frequencies between critically ill (H1N1)pdm09 Macedonian patients and healthy Macedonians reveals statistically significant difference for KIR2DL1 (frequency of 1 in the patients group, and 0.94 in the control group, $p=0.045$ ) (Table 1).

Linkage Disequilibrium. The classical linkage disequilibrium coefficient $(D)$, linkage disequilibrium coefficient $D$ standardized by the maximum value it can take $\left(D_{\max }\right)$, given the allele frequencies $\left(D^{\prime}\right)$, standardised simple measure of linkage disequilibrium $\left(r^{2}\right)$, and statistical significance $(P)$ for KIR genes are shown in Table 2. The genes present in all individuals (KIR2DL1, KIR2DL4, KIR3DL2, KIR3DL3, KIR2DP1 and KIR3DP1) were excluded from the analysis.

Pairs of KIR loci that displayed significant $(P<0.05)$ LD in critically ill Macedonian patients with pandemic influenza A (H1N1)pdm09 infection are given in Table 3. Positive LD was observed between pairs KIR3DL1 and KIR2DS4, KIR2DL2 with KIR2DS2 and KIR2DS3, KIR2DL5 and KIR3DS1, KIR3DS1 with KIR2DS1 and KIR2DS5, KIR2DS1 and KIR2DS5, and between KIR2DS2 with KIR2DS3. Negative LD was found between KIR3DL1 and KIR2DS5, KIR2DL3 and KIR2DS3, and KIR2DS4 and KIR2DS5. Other KIR genes were not in significant LD.

Genotype frequencies. KIR groups, genotype ID, KIR genotypes, number of individuals displaying certain genotype, and the frequency of genotypes are shown in Table 4.

If any of the genes 2DL2, 2DL5, 3DS1, 2DS1, 2DS2, 2DS3, or 2DS5 was present; the genotype was

Table 1: Comparison of the observed and estimated KIR gene frequencies for critically ill Macedonian patients with pandemic influenza $A(\mathrm{H} 1 \mathrm{~N} 1)$ pdm09 infection $(\mathrm{N}=63)$ and healthy Macedonians $(\mathrm{N}=214)$.

\begin{tabular}{|c|c|c|c|c|c|c|c|c|c|c|c|c|c|c|c|c|}
\hline & \multicolumn{16}{|c|}{ Frequencies for KIR genes in pandemic influenza A ( $\mathrm{H} 1 \mathrm{~N} 1)$ pd m09 infection in critically ill Macedonian patients and Macedonians } \\
\hline & \multicolumn{2}{|c|}{ Pseudogenes } & \multicolumn{8}{|c|}{ Inhibitory KIR } & \multicolumn{6}{|c|}{ Non inhibitory KIR } \\
\hline & \begin{tabular}{|c|}
$K I R$ \\
$2 D P 1$ \\
\end{tabular} & $\begin{array}{c}K I R \\
3 D P 1 \\
\end{array}$ & $\begin{array}{c}K I R \\
2 D L 1\end{array}$ & $\begin{array}{c}K I R \\
2 D L 2 \\
\end{array}$ & $\begin{array}{c}K I R \\
2 D L 3\end{array}$ & $\begin{array}{c}K I R \\
2 D L 4\end{array}$ & $\begin{array}{c}K I R \\
2 D L 5\end{array}$ & $\begin{array}{c}K I R \\
3 D L 1\end{array}$ & $\begin{array}{c}K I R \\
3 D L 2\end{array}$ & $\begin{array}{c}K I R \\
3 D L 3\end{array}$ & $\begin{array}{c}K I R \\
2 D S 1 \\
\end{array}$ & $\begin{array}{c}K I R \\
2 D S 2 \\
\end{array}$ & $\begin{array}{c}I R \\
2 D S 3 \\
\end{array}$ & $\begin{array}{c}K I R \\
2 D S 4\end{array}$ & $\begin{array}{c}K I R \\
2 D S 5\end{array}$ & $\begin{array}{c}K I R \\
3 D S 1\end{array}$ \\
\hline $\begin{array}{l}\text { H1N1 Infection } \\
\text { (N) }\end{array}$ & 63 & 63 & 63 & 36 & 56 & 63 & 27 & 60 & 63 & 63 & 29 & 32 & 26 & 60 & 20 & 30 \\
\hline $\begin{array}{l}\text { H1N1 Infection } \\
\text { (F) }\end{array}$ & 1 & 1 & 1 & 0.571 & 0.889 & 1 & 0.429 & 0.952 & 1 & 1 & 0.460 & 0.524 & 0.413 & 0.952 & 0.318 & 0.476 \\
\hline $\begin{array}{l}\text { H1N1 Infection } \\
\text { (GF) }\end{array}$ & 1 & 1 & 1 & 0.345 & 0.667 & 1 & 0.244 & 0.781 & 1 & 1 & 0.265 & 0.310 & 0.234 & 0.781 & 0.174 & 0.276 \\
\hline $\begin{array}{l}\text { Heal thy } \\
\text { Macedonians (N) }\end{array}$ & 210 & 214 & 201 & 126 & 192 & 214 & 89 & 201 & 214 & 214 & 103 & 122 & 77 & 201 & 64 & 84 \\
\hline $\begin{array}{l}\text { Heal thy } \\
\text { Macedonians (F) }\end{array}$ & 0.980 & 1 & 0.940 & 0.590 & 0.897 & 1 & 0.415 & 0.940 & 1 & 1 & 0.481 & 0.570 & 0.360 & 0.940 & 0.300 & 0.392 \\
\hline $\begin{array}{l}\text { Heal thy } \\
\text { Macedonians } \\
\text { (GF) }\end{array}$ & 0.870 & 1 & 0.760 & 0.360 & 0.690 & 1 & 0.230 & 0.800 & 1 & 1 & 0.280 & 0.350 & 0.180 & 0.800 & 0.170 & 0.220 \\
\hline Pearson's $p$ & 0.274 & $\&$ & 0.045 & 0.806 & 0.850 & $\&$ & 0.858 & 0.695 & $\&$ & $\&$ & 0.769 & 0.383 & 0.449 & 0.695 & 0.781 & 0.236 \\
\hline OR & $\&$ & $\&$ & $\&$ & 0.931 & 0.917 & $\&$ & 1.053 & 1.294 & $\&$ & $\&$ & 0.919 & 0.778 & 0.812 & 1.294 & 1.090 & 1.407 \\
\hline Wald $95 \% \mathrm{Cl}$ & \& & \& & \& & $\begin{array}{r}0.527- \\
1.644 \\
\end{array}$ & $\begin{array}{l}0.372- \\
2.257\end{array}$ & $\&$ & $\begin{array}{l}0.597- \\
1.859 \\
\end{array}$ & $\begin{array}{l}0.357- \\
4.690\end{array}$ & \& & \& & $\begin{array}{c}0.523- \\
1.615 \\
\end{array}$ & $\begin{array}{r}0.443- \\
1.367 \\
\end{array}$ & $\begin{array}{l}0.472- \\
1.395 \\
\end{array}$ & $\begin{array}{c}0.357- \\
4.690\end{array}$ & $\begin{array}{c}0.595- \\
1.998 \\
\end{array}$ & $\begin{array}{l}0.799- \\
2.477\end{array}$ \\
\hline
\end{tabular}

$\mathrm{N}$, number of individuals; $\mathrm{F}$, observed frequency was obtained by direct counting; GF, gene frequencies were calculated using the formula GF=1-Ö(1-F); p, statistical significance; \&, cannot be calculated because expected $<5, \mathrm{c} 2$ test; OR, Odds ratio; $\mathrm{Cl}$, confidence interval. 
Petlichkovski et al. Association of KIR Genes with Pandemic Influenza A (H1N1)pdm09 Infection

Table 2: LD analysis for KIR loci in critically ill Macedonian patients with pandemic influenza A (H1N1)pdm09 infection ( $\mathrm{N}=63)$ and in healthy Macedonians $(n=214)$.

\begin{tabular}{|c|c|c|c|c|c|c|c|c|c|c|c|c|}
\hline \multicolumn{13}{|c|}{ Pandemic influenza A (H1N1)pdm09 infection in critically ill Macedonian patients ( $N=63$ ) } \\
\hline & KIR3DL1 & KIR2DL1 & KIR2DL3 & KIR2DS4 & KIR2DL2 & KIR2DL5 & KIR3DS1 & KIR2DS1 & KIR2DS2 & KIRZDS3 & KIR2DS5 & $K I R 2 D P 1$ \\
\hline \multicolumn{13}{|c|}{ KIR3DL1 } \\
\hline$D^{a}$ & & \& & -0.0053 & 0.0454 & -0.0045 & -0.0113 & -0.0091 & -0.0257 & -0.0068 & 0.0038 & -0.0325 & \& \\
\hline$D_{2}^{\prime}$ & & \& & -1.0000 & 1.0000 & -0.2222 & -0.4167 & -0.3636 & -1.0000 & -0.3000 & 0.1923 & -1.0000 & \& \\
\hline$r^{2}$ & & \& & 0.0062 & 1.0000 & 0.0019 & 0.0116 & 0.0073 & 0.0586 & 0.0041 & 0.0013 & 0.1075 & $\&$ \\
\hline$P$ & & \& & 0.5303 & $<0.0001$ & 0.7327 & 0.3932 & 0.4985 & 0.0546 & 0.6117 & 0.7748 & 0.0093 & \& \\
\hline \multicolumn{13}{|c|}{$K I R 2 D L 1$} \\
\hline$D^{a}$ & 0.0156 & & $\&$ & $\&$ & \& & $\&$ & $\&$ & $\&$ & $\&$ & $\&$ & $\&$ & $\&$ \\
\hline$D^{\prime}$ & 0.3225 & & \& & \& & \& & \& & \& & \& & \& & \& & \& & \& \\
\hline$r^{2}$ & 0.0871 & & \& & \& & \& & \& & \& & \& & \& & \& & \& & \& \\
\hline$P$ & $<0.0001$ & & \& & $\&$ & $\&$ & $\&$ & $\&$ & \& & $\&$ & $\&$ & $\&$ & $\&$ \\
\hline \multicolumn{13}{|c|}{$K I R 2 D L 3$} \\
\hline$D$ & 0.0031 & 0.0134 & & -0.0053 & -0.0317 & -0.0317 & -0.0106 & 0.0035 & -0.0370 & -0.0494 & 0.0035 & $\&$ \\
\hline$D^{\prime}$ & 0.0569 & 0.2907 & & -1.0000 & -0.6667 & -0.5000 & -0.1818 & 0.0690 & -0.7000 & -0.7568 & 0.1000 & \& \\
\hline$r^{2}$ & 0.0018 & 0.0400 & & 0.0062 & 0.0417 & 0.0417 & 0.0045 & 0.0005 & 0.0557 & 0.1019 & 0.0006 & \& \\
\hline$P$ & 0.5318 & 0.0034 & & 0.5303 & 0.1052 & 0.1052 & 0.5926 & 0.8581 & 0.0611 & 0.0113 & 0.8482 & \& \\
\hline \multicolumn{13}{|c|}{ KIR2DS 4} \\
\hline$D$ & 0.0480 & 0.0158 & -0.0011 & & -0.0045 & -0.0113 & -0.0091 & -0.0257 & -0.0068 & 0.0038 & -0.0325 & $\&$ \\
\hline$D^{\prime}$ & 0.9113 & 0.3258 & -0.1894 & & -0.2222 & -0.4167 & -0.3636 & -1.0000 & -0.3000 & 0.1923 & -1.0000 & \& \\
\hline$r^{2}$ & 0.7628 & 0.0968 & 0.0002 & & 0.0019 & 0.0116 & 0.0073 & 0.0586 & 0.0041 & 0.0013 & 0.1075 & \& \\
\hline$P$ & $<0.0001$ & $<0.0001$ & 0.8192 & & 0.7327 & 0.3932 & 0.4985 & 0.0546 & 0.6117 & 0.7748 & 0.0093 & \& \\
\hline \multicolumn{13}{|c|}{$K I R 2 D L 2$} \\
\hline$D$ & -0.0110 & -0.0165 & -0.0423 & -0.0090 & & 0.0249 & -0.0023 & -0.0249 & 0.2245 & 0.0975 & -0.0068 & \& \\
\hline$D^{\prime}$ & -0.4388 & -0.7789 & -1.0000 & -0.3920 & & 0.1358 & -0.0101 & -0.1078 & 1.0000 & 0.5513 & -0.0375 & $\&$ \\
\hline$r^{2}$ & 0.0087 & 0.0230 & 0.0800 & 0.0064 & & 0.0104 & 0.0001 & 0.0102 & 0.8250 & 0.1602 & 0.0009 & \& \\
\hline$P$ & 0.1725 & 0.0266 & $<0.0001$ & 0.2427 & & 0.4188 & 0.9419 & 0.4222 & $<0.0001$ & 0.0015 & 0.8147 & \& \\
\hline \multicolumn{13}{|c|}{ KIR2DL5 } \\
\hline$D$ & -0.0215 & -0.0207 & -0.0227 & -0.0234 & 0.0822 & & 0.0658 & 0.0249 & 0.0454 & 0.0454 & 0.0385 & \& \\
\hline$D_{2}^{\prime}$ & -0.6049 & -0.6887 & -0.3775 & -0.7147 & 0.4808 & & 0.2929 & 0.1078 & 0.2222 & 0.1923 & 0.2125 & \& \\
\hline$r^{2}$ & 0.0332 & 0.0361 & 0.0229 & 0.0426 & 0.1150 & & 0.0708 & 0.0102 & 0.0337 & 0.0347 & 0.0280 & \& \\
\hline$P$ & 0.0077 & 0.0054 & 0.0268 & 0.0025 & $<0.0001$ & & 0.0347 & 0.4222 & 0.1453 & 0.1395 & 0.1841 & \& \\
\hline \multicolumn{13}{|c|}{ KIR3DS1 } \\
\hline$D$ & -0.0276 & -0.0172 & -0.0157 & -0.0294 & 0.0399 & 0.1311 & & 0.1459 & 0.0204 & 0.0098 & 0.1187 & $\&$ \\
\hline$D^{\prime}$ & -0.7467 & -0.5510 & -0.2517 & -0.8628 & 0.2473 & 0.5720 & & 0.6050 & 0.0900 & 0.0455 & 0.7136 & \& \\
\hline$r^{2}$ & 0.0558 & 0.0255 & 0.0112 & 0.0684 & 0.0276 & 0.2969 & & 0.3434 & 0.0067 & 0.0016 & 0.2606 & $\&$ \\
\hline$P$ & 0.0005 & 0.0196 & 0.1209 & 0.0001 & 0.0151 & $<0.0001$ & & $<0.0001$ & 0.5161 & 0.7511 & 0.0001 & \& \\
\hline \multicolumn{13}{|c|}{ KIR2DS1 } \\
\hline$D$ & -0.0315 & -0.0173 & -0.0113 & -0.0291 & 0.0390 & 0.1223 & 0.1522 & & -0.0030 & 0.0322 & 0.1713 & $\&$ \\
\hline$D^{\prime}$ & -1.0000 & -0.6495 & -0.2113 & -1.0000 & 0.1973 & 0.5668 & 0.7475 & & -0.0125 & 0.1448 & 1.0000 & \& \\
\hline$r^{2}$ & 0.0697 & 0.0246 & 0.0055 & 0.0640 & 0.0252 & 0.2465 & 0.3891 & & 0.0001 & 0.0173 & 0.5453 & \& \\
\hline$P$ & 0.0001 & 0.0217 & 0.2774 & 0.0002 & 0.0202 & $<0.0001$ & $<0.0001$ & & 0.9232 & 0.2969 & $<0.0001$ & \& \\
\hline KIR2DS & & & & & & & & & .0202 & & & \\
\hline$D$ & -0.0020 & -0.0081 & -0.0447 & 0.0316 & 0.2278 & 0.0304 & -0.0077 & 0.0198 & & 0.1172 & 0.0083 & $\&$ \\
\hline$D^{\prime}$ & -1.0000 & -1.0000 & -1.0000 & 0.1511 & 0.9799 & 0.1782 & -0.2920 & 0.1504 & & 0.5962 & 0.0550 & \& \\
\hline$r^{2}$ & 0.0036 & 0.0146 & 0.0881 & 0.0163 & 0.8725 & 0.0158 & 0.0042 & 0.0076 & & 0.2270 & 0.0013 & \& \\
\hline$P$ & 0.3795 & 0.0767 & $<0.0001$ & 0.0620 & $<0.0001$ & 0.0662 & 0.3409 & 0.2027 & & 0.0002 & 0.7765 & $\&$ \\
\hline KIR2DS & & & & & & & & & & & & \\
\hline$D$ & -0.0109 & 0.0091 & -0.0424 & -0.0079 & 0.1153 & 0.1074 & 0.0690 & 0.0605 & 0.0779 & & -0.0517 & $\&$ \\
\hline$D^{\prime}$ & -0.2791 & 0.4947 & -0.6450 & -0.2190 & 0.7789 & 0.5109 & 0.3159 & 0.3240 & 0.4312 & & -0.3942 & \& \\
\hline$r^{2}$ & 0.0090 & 0.0075 & 0.0848 & 0.0051 & 0.2382 & 0.2060 & 0.0868 & 0.0636 & 0.1017 & & 0.0508 & \& \\
\hline$P$ & 0.1661 & 0.2066 & $<0.0001$ & 0.2977 & $<0.0001$ & $<0.0001$ & $<0.0001$ & 0.0002 & $<0.0001$ & & 0.0736 & \& \\
\hline KIR2DS & & & & & & & & & & & & \\
\hline$D$ & -0.0330 & -0.0171 & -0.0108 & -0.0297 & 0.0314 & 0.1027 & 0.1284 & 0.1482 & 0.1190 & -0.0065 & & $\&$ \\
\hline$D^{\prime}$ & -0.7790 & -0.4777 & -0.1513 & -0.7606 & 0.2517 & 0.5786 & 0.6961 & 0.9407 & 0.7609 & -0.0593 & & \& \\
\hline$r^{2}$ & 0.0900 & 0.0283 & 0.0060 & 0.0788 & 0.0193 & 0.2051 & 0.3271 & 0.4160 & 0.2501 & 0.0009 & & $\&$ \\
\hline$P$ & $<0.0001$ & 0.0138 & 0.2566 & $<0.0001$ & 0.0421 & $<0.0001$ & $<0.0001$ & $<0.0001$ & $<0.0001$ & 0.6673 & & \& \\
\hline KIR2DP & & & & & & & & & & & & \\
\hline$D$ & -0.0011 & 0.0131 & 0.0168 & -0.0010 & -0.0077 & -0.0016 & -0.0067 & -0.0003 & -0.0447 & 0.0067 & -0.0037 & \\
\hline$D^{\prime}$ & -1.0000 & 0.7365 & 1.0000 & -1.0000 & -1.0000 & -0.1440 & -0.5885 & -0.0360 & -1.0000 & 1.0000 & -0.2819 & \\
\hline$r^{2}$ & 0.0012 & 0.1906 & 0.1662 & 0.0011 & 0.0133 & 0.0006 & 0.0102 & 0.0000 & 0.0881 & 0.0107 & 0.0035 & \\
\hline$P$ & 0.6076 & $<0.0001$ & $<0.0001$ & 0.6227 & 0.0916 & 0.7304 & 0.1394 & 0.9398 & $<0.0001$ & 0.1301 & 0.3889 & \\
\hline$K I R 3 D P$ & & & & & & & & & & & & \\
\hline$D$ & -0.0003 & -0.0002 & 0.0042 & -0.0003 & -0.0019 & 0.0019 & -0.0028 & 0.0022 & -0.0177 & 0.0017 & 0.0014 & 0.0046 \\
\hline$D_{2}^{\prime}$ & -1.0000 & -1.0000 & 1.0000 & -1.0000 & -1.0000 & 1.0000 & -1.0000 & 1.0000 & -0.7908 & 1.0000 & 1.0000 & 1.0000 \\
\hline$r^{2}$ & 0.0003 & 0.0003 & 0.0410 & 0.0003 & 0.0033 & 0.0033 & 0.0073 & 0.0044 & 0.0260 & 0.0026 & 0.0020 & 0.2465 \\
\hline$P$ & 0.7988 & 0.8155 & 0.0031 & 0.8070 & 0.4022 & 0.3977 & 0.2124 & 0.3344 & 0.0182 & 0.4524 & 0.5079 & $<0.0001$ \\
\hline
\end{tabular}

${ }^{a} D$, The classical linkage disequilibrium coefficient measuring deviation from random association between alleles at different loci; $D$ ', the linkage disequilibrium coefficient $D$ standardized by the maximum value it can take $\left(D_{\max }\right)$, given the allele frequencies; $r^{2}$, another way to standardise the simple measure of linkage disequilibrium [42]; $P$, statistical significance. \&, not calculated.

considered as B. If none of these were present, genotype is considered as $A A$. We have not attempted to distinguish between $A B$ and BB genotypes and called any of this Bx. $K I R$ genotypes were numerated according to the Allelefrequencies KIR Database [43]. Total of 29 different $K I R$ genotypes were found to be present in the studied sample, based on the presence of 16 KIR genes. We have found two AA genotypes, AA1 and AA180 with frequencies of 0.127 and 0.016 , respectively. The most frequent genotypes in the Bx group were genotypes Bx2 $(F=0.095), B \times 5(F=0.079)$ and $B \times 4(F=0.064)$. One new genotype of the $B x$ group was found and is being referred to Allelefrequencies.net (Table 4).
There is not statistically significant difference in distribution of AA and Bx KIR genotypes between Macedonian patients with (H1N1)pdm09 infection with severe course compared to healthy Macedonians $(P=0.207$, OR $=0.609$, Wald $95 \% \mathrm{Cl}=0.280-1.324)$ (Table 5).

\section{Discussion}

Influenza A has been an important threat to global public health and remains in the focus of clinical diagnosis, treatment, and basic research [44, 45]. While 
Table 3: Pairs of KIR loci that displayed significant $(p<0.05)$ LD in critically ill Macedonian patients with pandemic influenza $A$ (H1N1)pdm09 infection $(\mathrm{N}=63)$ and in healthy Macedonians $(n=214)$.

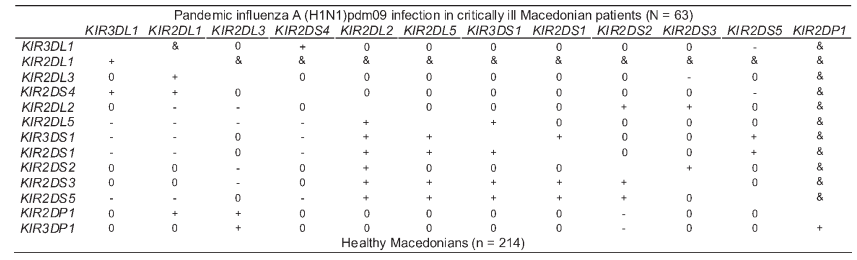

0, no significant LD; +, significant positive LD; -, significant negative LD; \&, LD not calculated.

hundreds of thousands of patients have been reported during the last outbreak of the (H1N1)pdm09 virus, we have witnessed that some subgroups of patients have a poorer outcome - the Mexico outbreak suggested that younger patients, especially those with co-morbidities and morbidly increased BMI were more susceptible to respiratory failure $[4,7]$. all in the control group $(P=0.001)$. Two other genotypes with significant differences in frequencies were $B \times 19$ $(P=0.012)$ and $B \times 63(P=0.045)$, both more frequent in the patients group. Comparable predominance of group Bx genotypes has been observed in many different populations, such as North Indians, Palestinians, South Asians, Afro-Caribbeans and also, general Macedonian population [46-49]. In a similar recent study analyzing the KIR polymorphisms in patients infected with Ebola virus [50], significantly higher frequencies of activating KIR2DS1 and KIR2DS3 genes were found in the group of fatally ill patients, when compared to the group of survivors from the infection. This finding led the authors to a conclusion that proposed overactivation of the NK cells was responsible for their rapid depletion. Although for the KIR2DS1 gene, we find "inverse" situation with these gene being more frequent in the control group, we still find this hypothesis tempting and possible, since as

Table 4: KIR locus haplogroups, genotypes ID and genotype frequency of critically ill Macedonian patients with pandemic influenza A (H1N1)pdm09 infection ( $N=63)$ and corresponding frequencies in healthy Macedonians $(n=214)$.

\begin{tabular}{|c|c|c|c|c|c|c|c|c|c|c|c|c|c|c|c|c|c|c|c|c|}
\hline $\begin{array}{l}\text { Haplo } \\
\text { group }\end{array}$ & $\begin{array}{c}\text { Genotype } \\
\text { ID }\end{array}$ & $\begin{array}{c}K I R \\
3 D L 1\end{array}$ & $\begin{array}{l}K I R \\
2 D L 1\end{array}$ & $\begin{array}{l}K I R \\
2 D L 3\end{array}$ & $\begin{array}{c}K I R \\
2 D S 4\end{array}$ & $\begin{array}{c}K I R \\
2 D L 2\end{array}$ & $\begin{array}{c}K I R \\
2 D L 5\end{array}$ & $\begin{array}{c}K I R \\
3 D S 1\end{array}$ & $\begin{array}{c}K I R \\
2 D S 1\end{array}$ & $\begin{array}{l}K I R \\
2 D S 2\end{array}$ & $\begin{array}{l}K I R \\
2 D S 3\end{array}$ & $\begin{array}{c}\text { KIR } \\
2 D S 5\end{array}$ & $\begin{array}{l}K I R \\
2 D L 4\end{array}$ & $\begin{array}{c}K I R \\
3 D L 2\end{array}$ & $\begin{array}{c}K I R \\
3 D L 3\end{array}$ & $\begin{array}{c}K I R \\
2 D P 1\end{array}$ & $\begin{array}{c}K I R \\
3 D P 1\end{array}$ & $\begin{array}{l}\text { Patients } \\
\text { No }(F)\end{array}$ & $\begin{array}{l}\text { Macedonians } \\
\text { No }(F)\end{array}$ & $\begin{array}{c}\text { Pearson's } \\
P\end{array}$ \\
\hline AA & 1 & 1 & 1 & 1 & 1 & 0 & 0 & 0 & 0 & 0 & 0 & 0 & 1 & 1 & 1 & 1 & 1 & $8(0,127)$ & $46(0,215)$ & 0,121 \\
\hline AA & 180 & 1 & 1 & 0 & 1 & 0 & 0 & 0 & 0 & 0 & 0 & 0 & 1 & 1 & 1 & 1 & 1 & $1(0,016)$ & 0 & 0,065 \\
\hline $\mathrm{Bx}$ & 2 & 1 & 1 & 1 & 1 & 0 & 1 & 1 & 1 & 0 & 0 & 1 & 1 & 1 & 1 & 1 & 1 & $6(0,095)$ & $11(0,051)$ & 0,203 \\
\hline $\mathrm{Bx}$ & 3 & 1 & 1 & 1 & 1 & 1 & 1 & 1 & 1 & 1 & 0 & 1 & 1 & 1 & 1 & 1 & 1 & $2(0,032)$ & $4(0,019)$ & 0,532 \\
\hline $\mathrm{Bx}$ & 4 & 1 & 1 & 1 & 1 & 1 & 0 & 0 & 0 & 1 & 0 & 0 & 1 & 1 & 1 & 1 & 1 & $4(0,064)$ & $27(0,126)$ & 0,461 \\
\hline $\mathrm{Bx}$ & 5 & 1 & 1 & 1 & 1 & 1 & 1 & 0 & 0 & 1 & 1 & 0 & 1 & 1 & 1 & 1 & 1 & $5(0,079)$ & $8(0,037)$ & 0,166 \\
\hline $\mathrm{Bx}$ & 8 & 1 & 1 & 1 & 1 & 0 & 1 & 1 & 1 & 0 & 1 & 0 & 1 & 1 & 1 & 1 & 1 & $1(0,016)$ & $2(0.009)$ & 0,660 \\
\hline $\mathrm{Bx}$ & 11 & 1 & 1 & 1 & 1 & 1 & 1 & 0 & 1 & 1 & 1 & 0 & 1 & 1 & 1 & 1 & 1 & $1(0,016)$ & $6(0,028)$ & 0,589 \\
\hline $\mathrm{Bx}$ & 13 & 1 & 1 & 1 & 1 & 1 & 1 & 1 & 0 & 1 & 1 & 0 & 1 & 1 & 1 & 1 & 1 & $1(0,016)$ & $6(0,028)$ & 0,589 \\
\hline $\mathrm{Bx}$ & 14 & 1 & 1 & 1 & 1 & 0 & 0 & 1 & 0 & 0 & 0 & 0 & 1 & 1 & 1 & 1 & 1 & $1(0,016)$ & $3(0,014)$ & 0,914 \\
\hline $\mathrm{Bx}$ & 19 & 1 & 1 & 1 & 1 & 1 & 0 & 0 & 0 & 0 & 0 & 0 & 1 & 1 & 1 & 1 & 1 & $3(0,048)$ & $1(0,005)$ & 0,012 \\
\hline $\mathrm{Bx}$ & 31 & 1 & 1 & 1 & 1 & 1 & 1 & 0 & 0 & 1 & 0 & 0 & 1 & 1 & 1 & 1 & 1 & $1(0,016)$ & 0 & 0,065 \\
\hline $\mathrm{Bx}$ & 62 & 1 & 1 & 1 & 1 & 1 & 0 & 0 & 0 & 1 & 1 & 0 & 1 & 1 & 1 & 1 & 1 & $3(0,048)$ & $3(0,014)$ & 0,107 \\
\hline $\mathrm{Bx}$ & 63 & 1 & 1 & 1 & 1 & 1 & 0 & 1 & 1 & 1 & 0 & 1 & 1 & 1 & 1 & 1 & 1 & $3(0,048)$ & $2(0,009)$ & 0,045 \\
\hline $\mathrm{Bx}$ & 68 & 0 & 1 & 1 & 0 & 1 & 1 & 1 & 1 & 1 & 0 & 1 & 1 & 1 & 1 & 1 & 1 & $1(0,016)$ & 0 & 0,065 \\
\hline $\mathrm{Bx}$ & 70 & 0 & 1 & 1 & 0 & 1 & 1 & 1 & 1 & 1 & 1 & 1 & 1 & 1 & 1 & 1 & 1 & $1(0,016)$ & $2(0,009)$ & 0,660 \\
\hline $\mathrm{Bx}$ & 90 & 1 & 1 & 0 & 1 & 1 & 1 & 1 & 1 & 1 & 1 & 0 & 1 & 1 & 1 & 1 & 1 & $1(0,016)$ & $2(0,009)$ & 0,668 \\
\hline $\mathrm{Bx}$ & 91 & 1 & 1 & 0 & 1 & 1 & 1 & 0 & 1 & 1 & 1 & 1 & 1 & 1 & 1 & 1 & 1 & $1(0,016)$ & 0 & 0,065 \\
\hline $\mathrm{Bx}$ & 94 & 1 & 1 & 0 & 1 & 1 & 1 & 1 & 0 & 1 & 1 & 0 & 1 & 1 & 1 & 1 & 1 & $3(0,048)$ & 0 & 0,001 \\
\hline $\mathrm{Bx}$ & 188 & 1 & 1 & 1 & 1 & 1 & 0 & 1 & 0 & 1 & 0 & 0 & 1 & 1 & 1 & 1 & 1 & $1(0,016)$ & 0 & 0,065 \\
\hline $\mathrm{Bx}$ & 200 & 1 & 1 & 1 & 1 & 0 & 1 & 0 & 0 & 0 & 0 & 0 & 1 & 1 & 1 & 1 & 1 & $2(0,032)$ & $1(0,005)$ & 0,068 \\
\hline $\mathrm{Bx}$ & 202 & 1 & 1 & 1 & 1 & 1 & 0 & 0 & 1 & 1 & 0 & 1 & 1 & 1 & 1 & 1 & 1 & $2(0,032)$ & $3(0,014)$ & 0,353 \\
\hline $\mathrm{Bx}$ & 205 & 1 & 1 & 1 & 1 & 0 & 0 & 0 & 1 & 0 & 1 & 0 & 1 & 1 & 1 & 1 & 1 & $2(0,032)$ & $1(0,005)$ & 0,068 \\
\hline $\mathrm{Bx}$ & 233 & 1 & 1 & 1 & 1 & 1 & 0 & 1 & 1 & 1 & 1 & 0 & 1 & 1 & 1 & 1 & 1 & $2(0,032)$ & $5(0,023)$ & 0,709 \\
\hline $\mathrm{Bx}$ & 319 & 1 & 1 & 1 & 1 & 1 & 0 & 1 & 1 & 1 & 1 & 1 & 1 & 1 & 1 & 1 & 1 & $2(0,032)$ & $2(0,009)$ & 0,190 \\
\hline $\mathrm{Bx}$ & 372 & 1 & 1 & 1 & 1 & 0 & 0 & 1 & 1 & 0 & 1 & 0 & 1 & 1 & 1 & 1 & 1 & $2(0,032)$ & $1(0,005)$ & 0,068 \\
\hline $\mathrm{Bx}$ & 375 & 0 & 1 & 1 & 0 & 0 & 0 & 0 & 1 & 0 & 0 & 1 & 1 & 1 & 1 & 1 & 1 & $1(0,016)$ & $1(0,005)$ & 0,356 \\
\hline $\mathrm{Bx}$ & 433 & 1 & 1 & 1 & 1 & 0 & 1 & 1 & 0 & 0 & 0 & 0 & 1 & 1 & 1 & 1 & 1 & $1(0,016)$ & 0 & 0,065 \\
\hline Bx & new & 1 & 1 & 0 & 1 & 1 & 0 & 0 & 1 & 1 & 1 & 1 & 1 & 1 & 1 & 1 & 1 & $1(0,016)$ & 0 & 0,065 \\
\hline
\end{tabular}

KIR Genotype [1=Positive, 0=negative]

We present the KIR genes distribution in Macedonian critically ill patients infected with (H1N1)pdm09. The studied group of patients and the healthy control subjects belonging to the same, Macedonian population used for comparison, showed similar frequencies for most KIR genes. The only statistically significant difference was noted for the inhibiting KIR2DL1 gene, which was present in all patients $(F=1)$ and in $94 \%$ of the controls $(P=0.045)$. Several statistically significant differences were found between the two populations when comparing the frequencies of $A A$ and Bx KIR genotypes, the most notable being for Bx94, which was present in 3 patients, but not present at much as four activating genes (KIR2DS3, KI2DS4, KIR2DS5 and KIR3DS1) were present in higher frequency (not statistically significant) in the critically ill

Table 5: Comparison of AA and Bx KIR haplogroup frequencies in critically ill Macedonian patients with pandemic influenza $A$ (H1N1)pdm09 infection $(N=63)$ and in healthy Macedonians $(n=214)$.

\begin{tabular}{|c|c|c|c|c|c|c|c|}
\hline \multirow[t]{2}{*}{ Haplogroup } & \multicolumn{2}{|c|}{$\begin{array}{l}\text { Pandemic influenza } \\
\text { A (H1N1)pdm09 } \\
\text { infection in critically } \\
\text { ill Macedonian } \\
\text { patients }\end{array}$} & \multicolumn{2}{|c|}{$\begin{array}{c}\text { Healthy } \\
\text { Macedonians }\end{array}$} & \multirow[t]{2}{*}{$\begin{array}{l}\text { Pearson's } \\
\text { p-value }\end{array}$} & \multirow[t]{2}{*}{$\begin{array}{l}\text { Odds } \\
\text { ratio }\end{array}$} & \multirow[t]{2}{*}{$\begin{array}{l}\text { Wald's } \\
95 \% \mathrm{Cl}\end{array}$} \\
\hline & $\mathrm{N}$ & $\mathrm{F}$ & $\mathrm{N}$ & $\mathrm{F}$ & & & \\
\hline $\begin{array}{l}\text { AA } \\
\text { Bx }\end{array}$ & $\begin{array}{c}9 \\
54 \\
\end{array}$ & $\begin{array}{l}14.3 \\
85.7\end{array}$ & $\begin{array}{c}46 \\
168 \\
\end{array}$ & $\begin{array}{l}21.5 \\
79.5\end{array}$ & 0.207 & 0.609 & $\begin{array}{l}0.280- \\
1.324\end{array}$ \\
\hline
\end{tabular}

$\mathrm{N}$, number of individuals displaying AA or Bx KIR genotype; $\mathrm{F}$, frequency of $K I R$ genotype; $\mathrm{Cl}$, confidence interval. 
patients when compared to the healthy subjects. This finding is in concordance with another recent study [51], where predominance of activating KIR genes (KIR2DS5 and KIR3DS1) was noted in severely ill patients with influenza (H1N1)pdm09 infection. It would be interesting to take into account the allelic polymorphism, which might allow different alleles to be expressed differentially and thus influence ligand binding and consecutive cytolysis [50]. Unfortunately, at present, we are not able to perform this analysis.

The results of tested linkage disequilibrium among KIR genes demonstrated that KIR genes present a wide range of linkage disequilibrium. Again, we cannot assume an absolute correlation between the KIR loci, as we only detect a certain percentage of alleles at a locus.

In conclusion, we have determined the distribution of KIR genes in patients with confirmed (H1N1)pdm09 infection and compared it to healthy control subjects, all living in Republic of Macedonia. While there is evident predominance of $K I R$ activating genes in the group of patients with very severe disease, these differences do not reach statistical significance. Our results suggest that activating KIR genes might be a predisposing factor for more severe viral disease and are in agreement with earlier proposed theories $[50,51]$. Our next step should be HLA genotyping of the samples in order to explore the ligands for the KIRs in the critically ill Macedonian patients with pandemic influenza $A$ (H1N1)pdm09 infection. Finally, KIR typing at allelic level might be more helpful in elucidating the different efficacy of NK cells and different disease course in virally infected patients.

\section{Acknowledgement}

The authors thank Elena Cvetkovska (Institute of Immunobiology and Human Genetics, Faculty of Medicine, University "Ss. Cyril i Methodius", Skopje, Republic of Macedonia) for isolation of genomic DNA, and taking care of the Macedonian Human DNA Bank (http://www.hdnamkd.org.mk).

\section{References}

1. World Health Organization (WHO). Pandemic (H1N1) 2009 - update 76, 2009. Available: http://www.who.int/csr/don/ 2009_11_27a/en/index.html. Accessed 2011 Nov 12.

2. World Health Organization (WHO). Pandemic (H1N1) 2009 - update 112, 2010. Available: http://www.who.int/csr/don/ 2010_08_06/en/index.html. Accessed 2011 Nov 19.

3. Chan M. World now at the start of 2009 inû uenza pandemic. World Health Organization, Geneva, Switzerland, 2009.

4. Novel Swine-Origin Influenza A (H1N1) Virus Investigation Team, Dawood FS, Jain S, Finelli L, Shaw MW, Lindstrom S, Garten RJ, Gubareva LV, Xu X, Bridges CB, Uyeki TM. Emergence of a novel swine-origin influenza $A(H 1 N 1)$ virus in humans. N Engl J Med. 2009;360(25):2605-15. Epub 2009 May 7. Erratum in: N Engl J Med. 2009;361(1):102.

5. Turner SJ, Doherty PC, Kelso A. Q\&A: H1N1 pandemic influenza—what's new? BMC Biol. 2010;8:130.

6. Xu R, Ekiert DC, Krause JC, Hai R, Crowe JE, Wilson IA. Structural basis of preexisting immunity to the $2009 \mathrm{H} 1 \mathrm{~N} 1$ pandemic influenza virus. Science. 2010;328(5976), 357-60.

7. Chowell G, Bertozzi SM, Colchero MA, Lopez-Gatell H, Alpuche-Aranda C, Hernandez M, Miller MA. Severe respiratory disease concurrent with the circulation of H1N1 influenza. N Engl J Med. 2009;361(7):674-9.

8. Fujisawa $\mathrm{H}$. Inhibitory role of neutrophils on influenza virus multiplication in the lungs of mice. Microbiol Immunol. 2001;45(10):679-88.

9. Hashimoto Y, Moki T, Takizawa T, Shiratsuchi A, Nakanishi Y. Evidence for phagocytosis of influenza virus-infected, apoptotic cells by neutrophils and macrophages in mice. J Immunol. 2007;178(4):2448-57.

10. Huber VC, Lynch JM, Bucher DJ, Le J, Metzger DW. Fc receptor-mediated phagocytosis makes a significant contribution to clearance of influenza virus infections. J Immunol. 2001;166(12):7381-8.

11. Kim HM, Lee YW, Lee KJ, Kim HS, Cho SW, van Rooijen $\mathrm{N}$, Guan Y, Seo SH. Alveolar macrophages are indispensable for controlling influenza viruses in lungs of pigs. J Virol. 2008;82(9):4265-74.

12. McGill J, Van Rooijen N, Legge KL. Protective influenzaspecific CD8 T cell responses require interactions with dendritic cells in the lungs. J Exp Med. 2008;205(7):1635-46.

13. Belz GT, Bedoui S, Kupresanin F, Carbone FR, Heath WR. Minimal activation of memory CD8+ T cell by tissue-derived dendritic cells favors the stimulation of naive CD8+ T cells. Nat Immunol. 2007;8(10):1060-6.

14. Mandelboim O, Lieberman N, Lev M, Paul L, Arnon TI, Bushkin Y, Davis DM, Strominger JL, Yewdell JW, Porgador A. Recognition of haemagglutinins on virus-infected cells by NKp46 activates lysis by human NK cells. Nature. 2001;409(6823):1055-60.

15. Stein-Streilein, J., Bennett, M., Mann, D., and Kumar, V. (1983). Natural killer cells in mouse lung: surface phenotype, target preference, and response to local influenza virus infection. J Immunol 131(6), 2699-704. 
16. Gazit, R., Gruda, R., Elboim, M., Arnon, T. I., Katz, G., Achdout, H., Hanna, J., Qimron, U., Landau, G., Greenbaum, E., Zakay-Rones, Z., Porgador, A., and Mandelboim, O. (2006). Lethal influenza infection in the absence of the natural killer cell receptor gene Ncr1. Nat Immunol 7(5), 517-23.

17. Ho, J. W., Hershkovitz, O., Peiris, M., Zilka, A., Bar-Ilan, A., Nal, B., Chu, K., Kudelko, M., Kam, Y. W., Achdout, H., Mandelboim, M., Altmeyer, R., Mandelboim, O., Bruzzone, R., and Porgador, A. (2008). H5-type influenza virus hemagglutinin is functionally recognized by the natural killer-activating receptor NKp44. J Virol 82(4), 2028-32.

18. Draghi, M., Pashine, A., Sanjanwala, B., Gendzekhadze, K., Cantoni, C., Cosman, D., Moretta, A., Valiante, N. M., and Parham, P. (2007). NKp46 and NKG2D recognition of infected dendritic cells is necessary for NK cell activation in the human response to influenza infection. J Immunol 178(5), 2688-98.

19. Liu, B., Mori, I., Hossain, M. J., Dong, L., Takeda, K., and Kimura, Y. (2004). Interleukin-18 improves the early defence system against influenza virus infection by augmenting natural killer cell-mediated cytotoxicity. J Gen Virol 85(Pt 2), 423-8.

20. Arnon, T. I., Achdout, H., Lieberman, N., Gazit, R., GonenGross, T., Katz, G., Bar-Ilan, A., Bloushtain, N., Lev, M., Joseph, A., Kedar, E., Porgador, A., and Mandelboim, O. (2004). The mechanisms controlling the recognition of tumor- and virusinfected cells by NKp46. Blood 103(2), 664-72.

21. Achdout, H., Meningher, T., Hirsh, S., Glasner, A., Bar-On, Y., Gur, C., Porgador, A., Mendelson, M., Mandelboim, M., and Mandelboim, O. Killing of avian and Swine influenza virus by natural killer cells. J Virol 84(8), 3993-4001.

22. Health Protection Agency; Health Protection Scotland; National Public Health Service for Wales; HPA Northern Ireland Swine influenza investigation teams (2009) Epidemiology of new influenza A (H1N1) virus infection, United Kingdom, AprilJune 2009. Euro Surveill 14, 19232.

23. Harel-Bellan, A., Quillet, A., Marchiol, C., DeMars, R., Tursz, T., and Fradelizi, D. (1986). Natural killer susceptibility of human cells may be regulated by genes in the HLA region on chromosome 6. Proc Natl Acad Sci U S A 83(15), 5688-92.

24. Moretta A, Tambussi G, Bottino C, Tripodi G, Merli A, Ciccone $E$, Pantaleo G, Moretta L. A novel surface antigen expressed by a subset of human CD3- CD16+ natural killer cells. Role in cell activation and regulation of cytolytic function. J Exp Med. 1990;171(3):695-714.

25. Liu WR, Kim J, Nwankwo C, Ashworth LK, Arm JP. Genomic organization of the human leukocyte immunoglobulin-like receptors within the leukocyte receptor complex on chromosome 19q13.4. Immunogenetics. 2000;51(8-9):659-69.

26. Gonzalez A, McErlean C, Meenagh A, Shovlin T, Middleton D. Killer cell immunoglobulin-like receptor allele discrimination by high-resolution melting. Hum Immunol. 2009;70(10):85863.
27. Uhrberg M, Valiante NM, Shum BP, Shilling HG, LienertWeidenbach K, Corliss B, Tyan D, Lanier LL, Parham P. Human diversity in killer cell inhibitory receptor genes. Immunity. 1997;7(6):753-63.

28. Middleton D, Gonzelez F. The extensive polymorphism of KIR genes. Immunology. 2010;129(1):8-19.

29. Khakoo SI, Thio CL, Martin MP, Brooks CR, Gao X, Astemborski J, Cheng J, Goedert JJ, Vlahov D, Hilgartner M, Cox S, Little AM, Alexander GJ, Cramp ME, O'Brien SJ, Rosenberg WM, Thomas DL, Carrington M. HLA and NK cell inhibitory receptor genes in resolving hepatitis $\mathrm{C}$ virus infection. Science. 2004;305(5685):872-4.

30. Jennes W, Verheyden S, Demanet C, Adjé-Touré CA, Vuylsteke B, Nkengasong JN, Kestens L. Cutting edge: resistance to HIV-1 infection among African female sex workers is associated with inhibitory KIR in the absence of their HLA ligands. J Immunol. 2006;177(10):6588-92.

31. Martin MP, Gao X, Lee JH, Nelson GW, Detels R, Goedert JJ, Buchbinder S, Hoots K, Vlahov D, Trowsdale J, Wilson M, O'Brien SJ, Carrington M. Epistatic interaction between KIR3DS1 and HLA-B delays the progression to AIDS. Nat Genet. 2002;31(4):429-34.

32. Jamil KM, Khakoo SI. KIR/HLA interactions and pathogen immunity. J Biomed Biotechnol. 2011;2011:298348.

33. Kulkarni S, Martin MP, Carrington M. The Yin and Yang of HLA and KIR in human disease. Semin Immunol. 2008;20(6):343-52.

34. Towner P. Purification of DNA. Essential Molecular Biology T. A. Brown. Oxford, Oxford University Press, 1995: 47-54.

35. Spiroski M, Arsov T, Petlichkovski A, Strezova A, Trajkov D, Efinska-Mladenovska O, Zaharieva E. Case Study: Macedonian Human DNA Bank (hDNAMKD) as a source for public health Genetics. Health Determinants in the Scope of New Public Health. B. G. Georgieva L. Sofia, Hans Jacobs Company, 2005:33-44.

36. Marsh SG, Parham P, Dupont B, Geraghty DE, Trowsdale J, Middleton D, Vilches C, Carrington M, Witt C, Guethlein LA, Shilling H, Garcia CA, Hsu KC, Wain H. Killer-cell immunoglobulin-like receptor (KIR) nomenclature report, 2002. Immunogenetics. 2003;55(4):220-6.

37. Robinson J, Waller MJ, Stoehr P, Marsh SG. IPD-the Immuno Polymorphism Database. Nucleic Acids Res. 2005;33(Database issue):D523-6.

38. Gómez-Lozano N, Vilches C. Genotyping of human killercell immunoglobulin-like receptor genes by polymerase chain reaction with sequence-specific primers: an update. Tissue Antigens. 2002;59(3):184-93. Erratum in: Tissue Antigens. 2004;64(5):629.

39. Excoffier L, Laval G, Schneider S. Arlequin (version 3.0): 
an integrated software package for population genetics data analysis. Evol Bioinform Online. 2007;1:47-50.

40. Mattiuz PL et al. New approaches to the population genetic and segregation analysis of the HLA system. In: Terasaki PI (ed) Histocompatibility testing. Munksgaard, Kopenhagen, 1971.

41. Lewontin RC, Kojima K. The evolutionary dynamics of complex polymorphisms. Evolution. 1960;14, 458-472.

42. Lewontin RC. The Interaction of Selection and Linkage. I. General Considerations; Heterotic Models. Genetics. 1964;49(1):49-67.

43. Middleton D, Menchaca L, Rood H, Komerofsky R. New allele frequency database: http://www.allelefrequencies.net. Tissue Antigens. 2003;61(5):403-7.

44. Yawata M, Yawata N, Draghi M, Little AM, Partheniou F, Parham P. Roles for HLA and KIR polymorphisms in natural killer cell repertoire selection and modulation of effector function. J Exp Med. 2006;203(3):633-45. Erratum in: J Exp Med. 2006;203(4):1131.

45. Nelson MI, Holmes EC. The evolution of epidemic influenza. Nat Rev Genet. 2007;8(3):196-205.

46. Cook MA, Moss PA, Briggs DC. The distribution of 13 killer-cell immunoglobulin-like receptor loci in UK blood donors from three ethnic groups. Eur J Immunogenet.
2003;30(3):213-21.

47. Norman PJ, Carrington CV, Byng M, Maxwell LD, Curran MD, Stephens HA, Chandanayingyong D, Verity DH, Hameed K, Ramdath DD, Vaughan RW. Natural killer cell immunoglobulin-like receptor (KIR) locus profiles in African and South Asian populations. Genes Immun. 2002;3(2):8695.

48. Rajalingam $R$, Krausa $P$, Shilling $H G$, Stein JB, Balamurugan A, McGinnis MD, Cheng NW, Mehra NK, Parham P. Distinctive KIR and HLA diversity in a panel of north Indian Hindus. Immunogenetics. 2002;53(12):1009-19.

49. Djulejic E, Petlichkovski A, Trajkov D, Hristomanova S, Middleton D, Spiroski M. Distribution of killer cell immunoglobulinlike receptors in the Macedonian population. Hum Immunol. 2010;71(3):281-8.

50. Wauquier N, Padilla C, Becquart P, Leroy E, Vieillard V. Association of KIR2DS1 and KIR2DS3 with fatal outcome in Ebola virus infection. Immunogenetics. 2010;62(11-12):76771.

51. Aranda-Romo S, Garcia-Sepulveda CA, Comas-Garcí a A, Lovato-Salas F, Salgado-Bustamante M, Gómez-Gómez A, Noyola DE. Killer-cell immunoglobulin-like receptors (KIR) in severe A (H1N1) 2009 influenza infections. Immunogenetics. 2012 Sep;64(9):653-62. 